\title{
Particle physics bounds from the Hulse-Taylor binary
}

\author{
Subhendra Mohanty* and Prafulla Kumar Panda ${ }^{\dagger}$ \\ Institute of Physics, Bhubaneswar-751005, India.
}

\begin{abstract}
The orbital period of the binary pulsar PSR $1913+16$ has been observed to decrease at the rate of $2.40 \times 10^{-12} \mathrm{~s} / \mathrm{s}$ which agrees with the prediction of the quadropole formula for gravitational radiation to within one percent. The decrease in orbital period may also occur by radiation of other massless particles like scalars and pseudoscalar Nambu-Goldstone bosons. Assuming that this energy loss is less than one percent of the gravitational radiation, we can establish bounds on couplings of these particles to nucleons. For a scalar nucleon coupling of the form $g_{s} \phi \bar{\psi} \psi$ we find that $g_{s}<3 \times 10^{-19}$. From the radiation loss of massless Goldstone bosons we establish the upper bound $\theta / f<7.5 \times 10^{-16} \mathrm{GeV}^{-1}$ on the QCD vacuum angle $\theta$ and the scale $f$ at which the baryon number symmetry can be broken spontaneously.
\end{abstract}

*mohanty@iopb.ernet.in

†prafulla@iopb.ernet.in 
The Hulse-Taylor (H-T) binary consisting of the pulsar PSR 1913+16 orbiting around an unseen companion star provides firm evidence for the existence of gravitational waves [1]. The observed loss of orbital period agrees with the prediction from the quadropole formula of gravitation radiation [2] to within one percent. In this letter we compute the orbital energy loss due to radiation of other massless particles like scalars and pseudoscalar Nambu-Goldstone bosons. Massless scalars which couple to nucleons arise in scalar-tensor theories of gravity [3], as dilatons in theories with spontaneously broken conformal symmetry [4] and in string theories [5]. Assuming a generic scalar-nucleon coupling $\mathcal{L}_{s}=g_{s} \phi_{s} \bar{\psi} \psi$ we find that the radiation of $\phi_{s}$ particles from the $\mathrm{H}-\mathrm{T}$ binary is less than $1 \%$ of the gravitational radiation loss if $g_{s}<3 \times 10^{-19}$. This gives an upper bound $\alpha_{B}=g_{s}^{2} / 4 \pi G m_{n}^{2} \leq 1$ on the ratio of a long range scalar mediated fifth force to the gravitational force between two nucleons. This bound is not as stringent as the bounds obtained from terrestrial fifth force search experiments [7] [8] which give $\alpha_{B}$ in the range of $10^{-3}-10^{-6}$.

In theories with a spontaneously broken global symmetry like the baryon number or the lepton number we have massless Nambu-Goldstone bosons (NGB) which have a generic coupling $\mathcal{L}_{p}=(m / f) \phi_{p} \bar{\psi} i \gamma_{5} \psi$ where $m$ is the fermion mass and $f$ is the scale of the global symmetry breaking [9]. The pseudoscalar field of a macroscopic source adds up coherently only if the spins of the constituents are polarised. It was observed by Chang, Mohapatra and Nussinov [10] and Barbieri et al [11] that the CP violating operator $\theta G \tilde{G}$ in the QCD sector induces a coupling $\mathcal{L}=(\theta / f)\left(m_{u} m_{d} /\left(m_{u}+m_{d}\right)\right) \phi_{p} \bar{\psi} \psi$ between the NGB $\phi_{p}$ and nucleons. This coupling give rise to a $1 / r$ type long range force and the NGB field of the constituent nucleons a macroscopic test body add up coherently even when their spins are randomly aligned. From the constraints on the energy carried away by the radiation of NGBs from the $\mathrm{H}-\mathrm{T}$ binary we obtain the upper bound $(\theta / f)<7.5 \times 10^{-16} \mathrm{GeV}^{-1}$. This can be compared with the separately established bounds $\theta<10^{-9}$ (from the measurement of the neutron electric dipole moment [12,13]) and $f>10^{8} \mathrm{GeV}$ (from the cooling rate of helium stars [14). The rate of energy loss by scalar particle emission is $\propto \Omega^{4}$ (where $\Omega$ is the orbital frequency). Observations of binary systems with faster orbital frequencies can be used to 
put more stringent bounds on couplings considered in this paper.

Finally we compute the energy loss by the radiation of neutrino pairs from the constituent neutrons of the H-T binary. We find that for the neutral current coupling $\mathcal{L}_{\nu}=(1 / \sqrt{2}) G_{F} n(x) \bar{\nu} \nu$, the energy radiated by neutrino pair emission is suppressed by the phase factor and is negligibly smaller than the gravitational radiation. Therefore if experimentally one observes a discrepancy between the observed period loss of the binary orbit and the prediction from gravitational radiation formula, it would be a signal of new kind of massless particle radiation and signal of new physics beyond the standard model.

Gravitational radiation : We use the Feynman rules of linearised quantum gravity [15 18] to compute the gravitational radiation from the Hulse-Taylor binary system. Assuming a universal graviton-matter coupling $\mathcal{L}_{I}=\sqrt{8 \pi G} h^{\mu \nu} T_{\mu \nu}$ we find that the energy loss by gravitational brehmstrahlung at tree level agrees with the Peter-Mathew expression [2] for classical gravitational wave radiation from binary system.

The effective Lagrangian for the graviton matter interaction is

$$
\mathcal{L}=-\frac{1}{4} \tilde{h}^{\mu \nu} \square \tilde{h}_{\mu \nu}+\kappa \tilde{h}^{\mu \nu} T_{\mu \nu}
$$

where the graviton field $h_{\mu \nu}$ is a perturbation of the metric $g_{\mu \nu}=\eta_{\mu \nu}+\kappa h_{\mu \nu}$ to the first order in $\kappa=\sqrt{8 \pi G}$. We have made the harmonic gauge choice, $\partial_{\mu} h_{\lambda}^{\mu}=(1 / 2) \partial_{\nu} h_{\mu}^{\mu}$ and defined $\tilde{h}_{\mu \nu}=h_{\mu \nu}-\frac{1}{2} \eta_{\mu \nu} h_{\lambda}^{\lambda}$ The universal coupling of $\tilde{h}^{\mu \nu}$ with matter at the tree level is a reflection of the equivalence principle of classical gravity. The rate of graviton emission is given by

$$
d \Gamma=\kappa^{2} \sum_{\lambda=1}^{2}\left|T_{\mu \nu}\left(k^{\prime}\right) \tilde{\epsilon}_{(\lambda)}^{\mu \nu}(k)\right|^{2} 2 \pi \delta\left(\omega-\omega^{\prime}\right) \frac{d^{3} k}{(2 \pi)^{3}} \frac{1}{2 \omega}
$$

where $T_{\mu \nu}\left(k^{\prime}\right)$ and $\tilde{\epsilon}_{\lambda}^{\mu \nu}(k)$ are the Fourier transforms of $T_{\mu \nu}(x)$ and $h^{\mu \nu}(x)$ and $\lambda$ denotes the polarisation of the emitted gravitons which is summed over. Using the harmonic gauge condition $k^{\mu}\left(\epsilon_{\mu \nu}-\frac{1}{2} \eta_{\mu \nu} \epsilon_{\lambda}^{\lambda}\right)=0$, we can set $\epsilon_{\mu 0}=0, \epsilon_{i}^{i}=0, k^{i} \epsilon_{i j}=0$ we have $\tilde{\epsilon}_{\mu \nu}=\epsilon_{\mu \nu}$ equation (2) can be written as

$$
d \Gamma=\frac{1}{2} \frac{\kappa^{2}}{(2 \pi)^{2}} \sum_{\lambda=1}^{2} \epsilon_{(\lambda)}^{i j}{ }^{*}(k) \epsilon_{(\lambda)}^{l m}(k) T_{i j}^{*}\left(\omega^{\prime}\right) T_{l m}\left(\omega^{\prime}\right) \omega \delta\left(\omega-\omega^{\prime}\right) d \omega d \Omega_{k}
$$


Using the relation

$$
\int d \Omega_{k} \sum_{\lambda=1}^{2} \epsilon_{(\lambda)}^{i j}{ }^{*}(k) \epsilon_{(\lambda)}^{l m}(k)=\frac{8 \pi}{5}\left[\frac{1}{2}\left(\delta_{i l} \delta_{j m}+\delta_{i m} \delta_{j l}\right)-\frac{1}{3} \delta_{i j} \delta_{l m}\right]
$$

We have the graviton emission rate

$$
d \Gamma=\frac{\kappa^{2}}{5 \pi}\left[T_{i j}\left(\omega^{\prime}\right) T_{j i}^{*}\left(\omega^{\prime}\right)-\frac{1}{3}\left|T^{i}{ }_{i}\left(\omega^{\prime}\right)\right|^{2}\right] \omega \delta\left(\omega-\omega^{\prime}\right) d \omega
$$

and the rate of energy loss by the graviton radiation is

$$
\frac{d E}{d t}=\int \frac{\kappa^{2}}{5 \pi} \omega^{2}\left[T_{i j}\left(\omega^{\prime}\right) T_{j i}^{*}\left(\omega^{\prime}\right)-\frac{1}{3}\left|T^{i}{ }_{i}\left(\omega^{\prime}\right)\right|^{2}\right] \delta\left(\omega-\omega^{\prime}\right) d \omega
$$

For a binary system of stars with masses $m_{1}, m_{2}$ in an eliptical orbit around the center of mass, the stress tensor is given by

$$
T_{\mu \nu}(x)=M \delta^{3}(\vec{x}-\vec{y}(t)) U_{\mu} U_{\nu}
$$

where $M=m_{1} m_{2} /\left(m_{1}+m_{2}\right)$ is the reduced mass and $U_{\mu}=\gamma(1, \dot{x}, \dot{y}, \dot{z})$ is the four velocity of the reduced mass in the elliptical orbit. Assuming an orbit in the $x-y$ plane with coordinates $\vec{y}(t)=(d \cos \theta, d \sin \theta)$, the relative distance $d$ and the angular velocity $\dot{\theta}$ are given by

$$
\begin{gathered}
d=\frac{a\left(1-e^{2}\right)}{1+e \cos \theta} \\
\dot{\theta}=\left[\frac{G\left(m_{1}+m_{2}\right) a\left(1-e^{2}\right)}{d^{2}}\right]^{1 / 2}
\end{gathered}
$$

where $a$ is the semimajor axis and $e$ the eccentricity of the eliptical orbit. Substitution (8) and (9) in (7) and taking the fourier transform we obtain the stress tensor $T_{i j}\left(\omega^{\prime}=n \Omega\right)$ for the Kepler orbit [2] in terms of the $n$ harmonics of the fundamental frequency $\Omega=$ $\left(G\left(m_{1}+m_{2}\right) a^{-3}\right)^{1 / 2}$,

$$
\begin{gathered}
T_{x x}\left(\omega^{\prime}\right)=-\frac{\omega^{\prime 2}}{4} M a^{2} \frac{2}{n}\left[J_{n-2}(n e)-2 e J_{n-1}(n e)+2 e J_{n+1}(n e)-J_{n+2}(n e)\right] \\
T_{y y}\left(\omega^{\prime}\right)=\frac{\omega^{\prime 2}}{4} M a^{2} \frac{2}{n}\left[J_{n-2}(n e)-2 e J_{n-1}(n e)+\frac{4}{n} J_{n}(n e)+2 e J_{n+1}(n e)-J_{n+2}(n e)\right]
\end{gathered}
$$




$$
T_{x y}\left(\omega^{\prime}\right)=\frac{\omega^{\prime 2}}{4 i} M a^{2} \frac{2}{n}\left(1-e^{2}\right)^{1 / 2}\left[J_{n-2}(n e)-2 J_{n}(n e)+J_{n+2}(n e)\right]
$$

From the above equation we obtain

$$
\left[T_{i j}\left(\omega^{\prime}\right) T_{j i}^{*}\left(\omega^{\prime}\right)-\frac{1}{3}\left|T^{i}{ }_{i}\left(\omega^{\prime}\right)\right|^{2}\right]=M^{2} a^{4} \omega^{\prime 4} g(n, e)
$$

where

$$
\begin{aligned}
g(n, e) & =\frac{1}{32 n^{2}}\left\{\left[J_{n-2}(n e)-2 e J_{n-1}(n e)+2 e J_{n+1}(n e)-J_{n+2}(n e)\right]^{2}\right. \\
& \left.+\left(1-e^{2}\right)\left[J_{n-2}(n e)-2 J_{n}(n e)+J_{n+2}(n e)\right]^{2}+\frac{4}{3 n^{2}}\left[J_{n}(n e)\right]^{2}\right\}
\end{aligned}
$$

Substituting (11) into (6) we have the rate of energy loss of a binary system in an elliptical orbit as sum of radiation in the $n$ harmonics of the fundamental frequency $\Omega$,

$$
\frac{d E}{d t}=\frac{32 G}{5} \sum_{n}(n \Omega)^{2} \cdot M^{2} a^{4}(n \Omega)^{4} g(n, e)
$$

Using the relation $\sum_{n}^{\infty} n^{6} g(n, e)=\left(1-e^{2}\right)^{-7 / 2}\left(1+\frac{73}{24} e^{2}+\frac{37}{96} e^{4}\right)$ [from [2]] and substituting the values of $\Omega=0.2251 \times 10^{-3} \mathrm{sec}^{-1}, m_{1}=1.42 M_{\odot}, m_{2}=1.4 M_{\odot}, a=3.0813815 \mathrm{lsec}$, $e=0.617127$ for the parameters of the H-T binary, we find that the energy loss is

$$
\begin{aligned}
\frac{d E}{d t} & =\frac{32}{5} \cdot G \cdot \Omega^{6} \cdot\left(\frac{m_{1} m_{2}}{m_{1}+m_{2}}\right)^{2} a^{4}\left(1-e^{2}\right)^{-7 / 2}\left(1+\frac{73}{24} e^{2}+\frac{37}{96} e^{4}\right) \\
& =3.2 \times 10^{33} \mathrm{erg} / \mathrm{sec}
\end{aligned}
$$

The time period of the elliptical orbit depends upon the energy $E$, so energy loss leads to a change of the time period of the orbit at the rate

$$
\begin{aligned}
\frac{d P_{b}}{d t} & =-6 \pi G^{-3 / 2}\left(m_{1} m_{2}\right)^{-1}\left(m_{1}+m_{2}\right)^{-1 / 2} a^{5 / 2}\left(\frac{d E}{d t}\right) \\
& =\frac{192 \pi}{5} G^{5 / 3} \Omega^{5 / 3} \frac{m_{1} m_{2}}{\left(m_{1}+m_{2}\right)^{1 / 3}}\left(1-e^{2}\right)^{-7 / 2}\left(1+\frac{73}{24} e^{2}+\frac{37}{96} e^{4}\right)
\end{aligned}
$$

which is the same expression as obtained by Peter and Mathews [2] for the classical gravitational radiation from binary systems. We find therefore that the tree level quantum gravity calculation agrees with the clasical results. For the Hulse - Taylor binary, the expression (15) yields the orbital period decceleration due to the gravitational radiation 
$\dot{P}_{b}=-2.403 \pm 0.002 \times 10^{-12}$ which agrees with the observed value from the Hulse - Taylor binary [1] $\dot{P}_{b}$ (observed $)=-2.40 \pm 0.09 \times 10^{-12}$ to with in $1 \%$. Energy loss by emission of other massless particles should be within $1 \%$ of energy loss by gravitational radiation and that can be used to put bounds on the couplings of the various massless scalar and pseudoscalar particles which arise in particle physics models.

Massless scalar radiation: We assume a coupling between massless scalar fields $\phi_{s}$ and the baryons of the form

$$
\mathcal{L}_{s}=g_{s} \phi_{s} \bar{\psi} \psi
$$

which for a macroscopic baryon source can be written as

$$
\mathcal{L}_{s}=g_{s} \phi_{s} n(x)
$$

where $n(x)$ is the baryon number density. A neutron star with radius $\sim 10 \mathrm{~km}$ can be regarded as a point source since the Compton wavelength of the radiation $\sim \Omega^{-1}=10^{9} \mathrm{~km}$ is much larger than the dimension of the source. The baryon number density $n(x)$ for the binary stars (denoted by $a=1,2$ ) may be written as

$$
n(x)=\sum_{a=1,2} N_{a} \delta^{3}\left(\vec{x}-\vec{x}_{a}(t)\right)
$$

where $N_{a} \sim 10^{57}$ is the total number of baryons in the neutron star and $\vec{x}_{a}(t)$ represents the Keplerian orbit of the binary stars. For the coupling (17) and the source (18) the rate of scalar particles emitted from the neutron star in orbit with frequency $\Omega$ is

$$
d \Gamma=|n(\omega)|^{2}(2 \pi) \delta\left(\omega-\omega^{\prime}\right) \frac{d^{3} \omega^{\prime}}{(2 \pi)^{3} 2 \omega^{\prime}}
$$

the rate of energy loss by massless scalar radiation is

$$
\frac{d E}{d t}=\int|n(\omega)|^{2} \omega^{\prime}(2 \pi) \delta\left(\omega-\omega^{\prime}\right) \frac{d^{3} \omega^{\prime}}{(2 \pi)^{3} 2 \omega^{\prime}}
$$

where $n(\omega)$ is the fourier expansion of the source density (18)

$$
n(\omega)=\frac{1}{2 \pi} \int e^{i \vec{k} \cdot \vec{x}} e^{-i \omega t} \sum_{a=1,2} N_{a} \delta^{3}\left(\vec{x}-\vec{x}_{a}(t)\right) d^{3} x d t
$$


with $\omega=n \Omega$. Going over to the c.m. coordinates $\vec{r}=(x, y)$ by substituting $\vec{x}_{1}=m_{2} \vec{r} /\left(m_{1}+m_{2}\right), \vec{x}_{2}=-m_{1} \vec{r} /\left(m_{1}+m_{2}\right)$ we have

$$
n(\omega)=\left(N_{1}+N_{2}\right) \delta(\omega)+\left(\frac{N_{1}}{m_{1}}-\frac{N_{2}}{m_{2}}\right) M\left(i k_{x} x(\omega)+i k_{y} y(\omega)\right)+O(\vec{k}, \vec{r})^{2}
$$

where $(x(\omega), y(\omega))$ are the fourier components of the Kepler orbit of the reduced mass in the c.m. frame given by (8)-(9). The first term in $n(\omega)$ is a delta function which has vanishing contribution to (20). The leading non-zero contribution comes from the second term in (22). Substituting [6]

$$
x(\omega)=\frac{2 a}{n} J_{n}^{\prime}(n e), \quad y(\omega)=\frac{2 i a \sqrt{1-e}}{n e} J_{n}(n e)
$$

in (22) we obtain the expression for $|n(\omega)|^{2}$ given by

$$
|n(\omega)|^{2}=\frac{4}{3}\left[\left(\frac{N_{1}}{m_{1}}-\frac{N_{2}}{m_{2}}\right) M\right]^{2} a^{2} \Omega^{2}\left[{J_{n}^{\prime 2}}_{n}^{2}(n e)+\frac{\left(1-e^{2}\right)}{e^{2}} J_{n}^{2}(n e)\right]
$$

where we have used the dispersion relation $k_{x}^{2}=k_{y}^{2}=\frac{1}{3}(n \Omega)^{2}$. Substituting (24) in (20) we have the rate of energy loss by massless scalars

$$
\frac{d E}{d t}=\frac{2}{3 \pi}\left[\left(\frac{N_{1}}{m_{1}}-\frac{N_{2}}{m_{2}}\right) M g_{s}\right]^{2} a^{2} \Omega^{4} \sum_{n} n^{2}\left[J_{n}^{\prime 2}(n e)+\frac{\left(1-e^{2}\right)}{e^{2}} J_{n}^{2}(n e)\right]
$$

The mode sum can be carried out using the Bessels function series formulas given in reference [2] to give $\sum_{n} n^{2}\left[J_{n}^{\prime 2}(n e)+\left(1-e^{2}\right) e^{-2} J_{n}^{2}(n e)\right]=(1 / 4)\left(2+e^{2}\right)\left(1-e^{2}\right)^{-5 / 2}$. The energy loss (25) in terms of the orbital parameters $\Omega, a$ and $e$ is given by

$$
\frac{d E}{d t}=\frac{1}{3 \pi}\left[\left(\frac{N_{1}}{m_{1}}-\frac{N_{2}}{m_{2}}\right) M g_{s}\right]^{2} \Omega^{4} a^{2} \frac{\left(1+e^{2} / 2\right)}{\left(1-e^{2}\right)^{5 / 2}}
$$

Since $N_{a} m_{n}=m_{a}-\epsilon_{a}$ where $\epsilon_{a}=\frac{G m_{a}^{2}}{R_{a}}$ is the gravitational binding energy and $m_{n}$ the neutron mass, the factor $\left(\frac{N_{1}}{m_{1}}-\frac{N_{2}}{m_{2}}\right)=G\left(\frac{m_{1}}{R_{1}}-\frac{m_{2}}{R_{2}}\right)$. For the H-T binary $m_{1}-m_{2} \simeq 0.02 M_{\odot}$ and $R_{a} \sim 10 \mathrm{~km}$, therefore $\left(\frac{N_{1}}{m_{1}}-\frac{N_{2}}{m_{2}}\right) \simeq 3 \times 10^{-3} \mathrm{GeV}^{-1}$.

For the H-T binary the rate of energy loss terms out to be

$$
\frac{d E}{d t}=g_{s}^{2} \times 9.62 \times 10^{67} \mathrm{ergs} / \mathrm{sec}
$$


Assuming that this is less than $1 \%$ of the gravitational energy loss i.e. $d E / d t \leq 10^{31}$ ergs/sec, we obtain gives an upper bound on scalar nucleon coupling

$$
g_{s}<3 \times 10^{-19} \text {. }
$$

Exchange of massless scalar between two nucleons gives rise to spin independent fifth force with the static potential $V_{s s}(\vec{r})=-g_{s}^{2} / 4 \pi r$, which shows that ratio of the fifth force to the gravitational force between two nucleons is $\alpha_{B}=\frac{g_{s}^{2}}{4 \pi G m_{n}^{2}} \leq 1$. This is less stringent than the bound $\alpha_{B} \sim 10^{-3}-10^{-6}$ obtained from terrestrial fifth force search experiments [7 9 . Nambu-Goldstone boson radiation: Massless pseudoscalar particles arise as NambuGoldstone bosons (NGB) when some global symmetry is broken spontaneously [19 21]. We consider the coupling of massless NGBs $\phi_{p}$ to baryons,

$$
\mathcal{L}_{p}=\frac{1}{f}\left(\partial_{\mu} \phi_{p}\right) \bar{\psi} \gamma^{\mu} \gamma^{5} \psi=\frac{m}{f} \phi_{p}\left(\bar{\psi} i \gamma_{5} \psi\right)
$$

(where the last equality holds for fermions on mass shell) which arise in many particle physics models where a global symmetry is broken spontaneously at some scale $f$ giving rise to Nambu-Goldstone bosons. This coupling gives rise to a spin dependent long-range force [22] $V(r) \sim(1 / f)^{2}\left(1 / r^{3}\right)\left(\sigma_{1} \cdot \sigma_{2}-3\left(\sigma_{1} \cdot \hat{r}\right)\left(\sigma_{2} \cdot \hat{r}\right)\right)$. The pseudoscalar coupling in the first order in $g_{p}$ is spin dependent and the field of a macroscopic body with randomly oriented constituents averages to zero. The radiation of NGB by the $N$ constituent particles of the macroscopic system will be in coherent which means that the energy radiated by $N$ particles will be $N$ times the single particle energy loss. This is different from scalar radiation which being coherent scales as $N^{2}$ times the single particle radiation.

It was observed by Chang, Mohapatra and Nussinov [10] and Barbieri et al [11] that if there is $\mathrm{CP}$ violation in the theory then $\phi_{p}$ can have both pseudoscalar coupling as in (29) as well as scalar coupling of the form (16). For example the CP violating QCD term $\theta G \tilde{G}$ will induce an interaction between $\phi_{p}$ and nucleons of the form

$$
\mathcal{L}_{p}=\frac{\theta}{f}\left(\frac{m_{u} m_{d}}{m_{u}+m_{d}}\right) \phi_{p} \bar{\psi} \psi
$$


This scalar coupling gives rise to a long range $(V(r) \sim 1 / r)$ potential which is spin independent so that $\phi_{p}$ field outside a macroscopic object adds up coherently. The form of the interaction term (30) is the same as in equation (16) so the same bound (28) holds for the dimensionless coupling

$$
\frac{\theta}{f}\left(\frac{m_{u} m_{d}}{m_{u}+m_{d}}\right)<3 \times 10^{-19}
$$

which means that for $m_{u}=5 \mathrm{MeV}, m_{d}=9 \mathrm{MeV}$ we have $\theta / f<7.5 \times 10^{-16} \mathrm{GeV}^{-1}$. This can be compared with the separate bounds $\theta<10^{-9}$ (obtained from neutron edm [12,13]) and $f>10^{8} \mathrm{GeV}$ (obtained from the cooling rate of helium stars [14). The bound (31) holds for models where the mass of the Nambu-Goldstone boson is smaller than the frequency $\Omega \simeq 10^{-19} \mathrm{eV}$ of the binary orbit. In axion models [23 the axion acquires a mass due to QCD instantons which ranges between $10^{-3} \mathrm{eV}-10^{-6} \mathrm{eV}$, so our bound does not hold for these model. In majorons models [19,20] where the lepton number is broken spontaneously, the majorons which arise as NGBs can remain massless and our bound would then hold. Neutrino radiation: The coupling of neutrinos to neutrons in the standard model is via the weak neutral current and is given by

$$
\mathcal{L}_{\nu}=\sqrt{2} G_{F} \frac{n(x)}{2} \bar{\nu}_{L} \gamma^{0} \nu_{L}
$$

where $n(x)$ is the number density of the neutrons which are the source of the neutrino field. The radiation of neutrinos from the $\mathrm{H}$-T binary with $n(x)=N \delta^{3}(x-x(t))$ carries aways energy at the rate

$$
\frac{d E}{d t} \simeq\left(\frac{G_{F}^{2} N^{2}}{4}\right) \Omega^{6}=10^{-43} \mathrm{ergs} / \mathrm{sec} .
$$

The weak coupling of neutrinos to neutrons is much larger than the coupling of the gravitons to matter, however since the emission of neutrinos occurs in pairs, the phase space suppression makes the energy radiated by neutrino emission negligible compared to graviational radiation.

The period loss in the $\mathrm{H}-\mathrm{T}$ system has been determined by measuring the time of periastron over a period of almost 19 years. The accuracy of the measured value of period 
loss increases quadratically with time. If in the course of observation one finds a significant discrepancy between the observed value of period loss and the prediction of the gravitational quadropole formula, it would be a compelling signal of physics beyond standard model. Note added: Astrophysical bounds from the Hulse-Taylor binary have been considered in references [24] and [25]. In [24] the energy loss in Brans-Dicke gravity is derived and in [25] effect of scalar couplings on orbital parameters of the H-T binary is studied. 


\section{REFERENCES}

[1] R.A. Hulse and J.H. Taylor, Ap. J. Lett 195, L51 (1975); J.H. Taylor and J.M. Weisberg, Ap. J. 253, 908 (1982); J.M. Weisberg and J.H. Taylor, Phys. Rev. Lett. 52, 1348 (1984).

[2] P.C. Peters and J. Mathews, Phys. Rev. 131, 435 (1963).

[3] C.H. Brans and R.H. Dicke, Phys. Rev. 124, 925 (1961); R.H. Dicke, Phys. Rev. 125, 2163 (1962); S. Weinberg, Gravitation and Cosmology, John Wiley, (1972).

[4] Y. Fujii, Nature, 2345 (1971).

[5] M.B. Green, J.H. Schwarz and E. Witten, Superstring Theory Vol. II, Cambridge University Press (1987).

[6] L.D. Landau and E.M. Lifshitz, Classical Theory of Fields, Pergamon Press.

[7] Adelberger et al Phys. Rev. D42, 3267 (1990); Ann. Rev. Nucl. Part. Sci. 41269 (1991).

[8] C.W. Stubbs et al, Phys. Rev. Lett. 62, 609 (1989); B.R. Heckel et al Phys. Rev. Lett. 63, 2705 (1989); R. Cowsik, N. Krishnan, S.N. Tandon, C.S. Unikrishnan, Phys. Rev. Lett 64, 336 (1990); R. Cowsik et al TIFR preprint (1993).

[9] R. Mohapatra, Unification and Supersymmetry, Springer-Verlag (1986).

[10] D. Chang, R. Mohapatra, S. Nussinov, Phys. Rev. Lett. 55, 2835 (1985).

[11] R. Barbieri, R.N. Mohapatra, D. Nanopoulous and D. Wyler, Phys. Lett. B107, 80 (1981).

[12] I.S. Alterv, et al JETPL 44, 460 (1986); J.M. Pendlebury et al Phys. Lett. B136, 327 (1984).

[13] V. Baluni, Phys. Rev. D19, 2227 (1979); R.J. Crewther, et al Phys. Lett. B88 123 (1979).

[14] D. Dicus, E. Kolb, V.T. Teplitz and R. Wagener, Phys. Rev. D10, 1829 (1979); M. 
Fukugita, W. Watamura and M. Yoshimura, Phys. Rev. Lett. 48, 1522 (1982); G.G. Raffelt and D.S.P. Dearborn, Phys. Rev. D36, 2211 (1987).

[15] Feynman Lectures on gravitation, Caltech notes (1962)

[16] T.W.B. Kibble in High energy Physics IAEA Vienna, 885 (1965).

[17] C.J. Isham etal Quantum Gravity, Oxford (1975).

[18] D. Boulaware and S. Deser, Ann. Phys. (NY) 89, 193 (1975).

[19] Y. Chikashige, R.N. Mohapatra and R.D. Pecci, Phys. Lett. B98, 265 (1981).

[20] G. Gelmini and M. Roncadelli, Phys. Lett. B99, 411 (1981); C.S. Aulakh and R.N. Mohapatra, Phys. Lett. B119 136 (1983); S. Bertolini and A. Santamaria, Nucl. Phys. B310, 714 (1988).

[21] G. Gelmini, S. Nussinov and T. Yanagida, Nucl. Phys. B219, 31 (1983); H. Georrgi, S. L. Glashow and S. Nussinov Nucl. Phys. B193, 297 (1981); R. Barbieri and R.N. Mohapatra, Z. Phys. C11, 175 (1981); F. Wilczek, Phys. Rev. Lett. 49, 1549 (1982); D. Reiss, Phys. Lett. 115, 217 (1982).

[22] J. Moody and F. Wilczek, Phys. Rev. D30, 130 (1984); G. Feinberg and J. Sucher, Phys. Rev. D20, 1717 (1979).

[23] R.D. Peccei, H. Quinn, Phys. Rev. Lett. 38, 1440 (1977); S. Weinberg, Phys. Rev. Lett. 40, 223 (1978); F. Wilczek, Phys. Rev. Lett. 40, 279 (1979); J.E. Kim, Phys. Rep. 150, $1(1987)$.

[24] C.M. Will and H.W. Zaglauer, Ap. J. 346, 366 (1989).

[25] C.P. Burgess and J. Cloutier, Phys. Rev. D38, 2944 (1988). 